The Astrophysical Journal, 483:98-102, 1997 July 1

(C) 1997. The American Astronomical Society. All rights reserved. Printed in U.S.A.

\title{
MEASURING THE ROTATION SPEED OF GIANT STARS FROM GRAVITATIONAL MICROLENSING
}

\author{
ANDREW GOULD ${ }^{1}$ \\ Department of Astronomy, Ohio State University, Columbus, OH 43210; gould@payne.mps.ohio-state.edu \\ Received 1996 November 12; accepted 1997 January 23
}

\begin{abstract}
During some gravitational lensing events, the lens transits the disk of the star. This event causes a shift in the apparent radial velocity of the star proportional to the star's rotation speed. The magnification of such an event is different from that expected for a point source. By measuring both effects, one can determine the rotation parameter $v \sin i$. The method is especially useful for $\mathrm{K}$-giant stars because these stars have turbulent velocities that are typically large compared with their rotation speed. By making a series of radial velocity measurements, one can typically determine $v$ sin $i$ to the same accuracy as the individual radial velocity measurements. There are approximately 10 microlensing transit events per year that would be suitable for these measurements.
\end{abstract}

Subject headings: gravitational lensing — stars: late-type — stars: rotation

\section{INTRODUCTION}

There are two principal methods of determining the rotation speed $v$ of a star. First, one can measure $v$ sin $i$ spectroscopically from the broadening of the spectral lines, where $i$ is the angle between the line of sight and the spin axis of the star. For giant stars, however, line broadening due to turbulence is typically $4-8 \mathrm{~km} \mathrm{~s}^{-1}$ (Gray 1989). Hence the small additional broadening we expect to arise from rotation is difficult to detect. For G giants, Gray (1989) clearly detected $v \sin i$ of order 5 $\mathrm{km} \mathrm{s}^{-1}$ with errors of $\sim 1 \mathrm{~km} \mathrm{~s}^{-1}$, but $\mathrm{K}$ giants rotate more slowly, and for these stars the detections are marginal. Second, one can measure the rotation period $P$ photometrically from the periodic variation in luminosity due to starspots. Then from the known (or assumed) radius $r$, one finds the rotation speed $v=2 \pi r / P$. The Optical Gravitational Lensing Experiment group has found that $O(1 \%)$ of giant stars seen toward Baade's Window have spots of sufficient amplitude to allow measurement of their rotation periods (Olech 1997). However, the rotation speeds of these chromospherically active giant stars may not be representative of the population as a whole.

Here I present an alternate method of measuring the $v \sin i$ of giant stars by making spectroscopic and photometric observations of a subclass of ongoing microlensing events. Microlensing occurs when the projected separation $\theta$ between a foreground object (the lens) and a more distant source star is of order the Einstein radius $\theta_{\mathrm{E}}$,

$$
\theta_{\mathrm{E}}^{2} \equiv \frac{4 G M d_{\mathrm{ls}}}{c^{2} d_{\mathrm{ol}} d_{\mathrm{os}}}
$$

where $M$ is the mass of the lens and $d_{\mathrm{ol}}, d_{1 \mathrm{~s}}$, and $d_{\mathrm{os}}$ are the distances between the observer, the lens, and the source. The lens then magnifies the source by an amount $A(x)$,

$$
A(x)=\frac{x^{2}+2}{x\left(x^{2}+4\right)^{1 / 2}}, \quad x \equiv \frac{\theta}{\theta_{\mathrm{E}}} .
$$

Let $\theta_{*}$ be the angular radius of the source. If the lens comes sufficiently close to the source, $\theta \lesssim \theta_{*}$, the different parts of the source will be magnified by significantly different amounts. This differential magnification leads to two different kinds of effects. First, the total magnification begins to deviate from the simple point source formula $A(x)$, which allows one to measure $x_{*}$, where

$$
x_{*} \equiv \frac{\theta_{*}}{\theta_{\mathrm{E}}},
$$

the stellar radius in units of the Einstein radius (Gould 1994; Nemiroff \& Wickramasinghe 1994; Witt \& Mao 1994). Second, if the star is spinning, then the side that is moving toward us will in general be closer to (or farther from) the lens than the side that is moving away from us. Since this side is magnified more, the centroids of the stellar lines will be shifted to the blue (or the red). From this shift one can measure the quantity,

$$
U \equiv x_{*} v \sin i
$$

provided that the lens passes within a few stellar radii of the source (Maoz \& Gould 1994).

Heretofore, the main interest in these effects was that they allowed measurement of $\theta_{\mathrm{E}}$. If $x_{*}$ is measured, and if the angular size of the source star is known (as it usually is, from Stefan's law, flux $\propto T^{4} \theta_{*}^{2}$ ), one can determine $\theta_{\mathrm{E}}=\theta_{*} / x_{*}$. If both the

${ }^{1}$ Alfred P. Sloan Foundation Fellow. 
angular size and the projected rotation speed are known (as they might be for A stars observed in the Large Magellanic Cloud), $\theta_{\mathrm{E}}=U^{-1} \theta_{*} v \sin i$.

Here I point out that by combining photometric and spectroscopic measurements, one can determine $x_{*}$ and $v$ sin $i$ separately. I estimate that it may be possible to use microlensing to determine $v \sin i$ for about 10 giants per year.

\section{MICROLENSING AT CLOSE QUARTERS}

Three groups have been monitoring a total of several $10^{7}$ stars toward the Galactic bulge (Alcock et al. 1995; Udalski et al. 1994; Alard 1996). Together they have observed more than 100 microlensing events. A fourth group (Aubourg et al. 1993; Ansari 1996) has recently joined this search. If these searches were tuned primarily to finding lensing events of bulge giants, events could be found at a rate of $\sim 170 \mathrm{yr}^{-1}$, including $\sim 10 \mathrm{yr}^{-1}$ in which the lens transits the disk of the star, i.e., events for which $\theta<\theta_{*}$ (or $x<x_{*}$ ) at the peak (Gould 1995b). It is these transit events that provide the main opportunity to measure $v$ $\sin i$.

The finite size of the source causes the light curve to deviate from its standard form according to

$$
A(x) \rightarrow A(x) B_{0}(z), \quad z \equiv \frac{\theta}{\theta_{*}},
$$

where $x$ is now regarded as the separation between the lens and the center of the star and $B_{0}(z)$ is the function shown in Figure 1 (Gould 1994). Because of the structure of this curve at $z \sim 1$, it is quite easy to measure $x_{*}$ whenever $z$ reaches a minimum value $z_{\min } \lesssim 1$. (Note from Fig. 1 that the deviation is significant even for $z \sim 2$. However, a more detailed analysis shows that with single-band photometry it is not possible to measure $x_{*}$ unless $z \lesssim 1$. Such measurements are possible up to $z \sim 2$ using two-band optical/infrared photometry [Gould \& Welch 1996].)

In computing $B_{0}(z)$ I have assumed that the star is limb darkened, with surface brightness

$$
S(z)=S(0)\left[1-\kappa_{1} Y-\kappa_{2} Y^{2}\right], \quad Y \equiv 1-\left(1-z^{2}\right)^{1 / 2},
$$

and have adopted parameters $\kappa_{1}=0.567$ and $\kappa_{2}=0.114$ suitable for a star at $T=4500 \mathrm{~K}$ observed in the $I$ band. As I discuss in $\S 4$, uncertainties in the limb-darkening coefficients play a very small role.

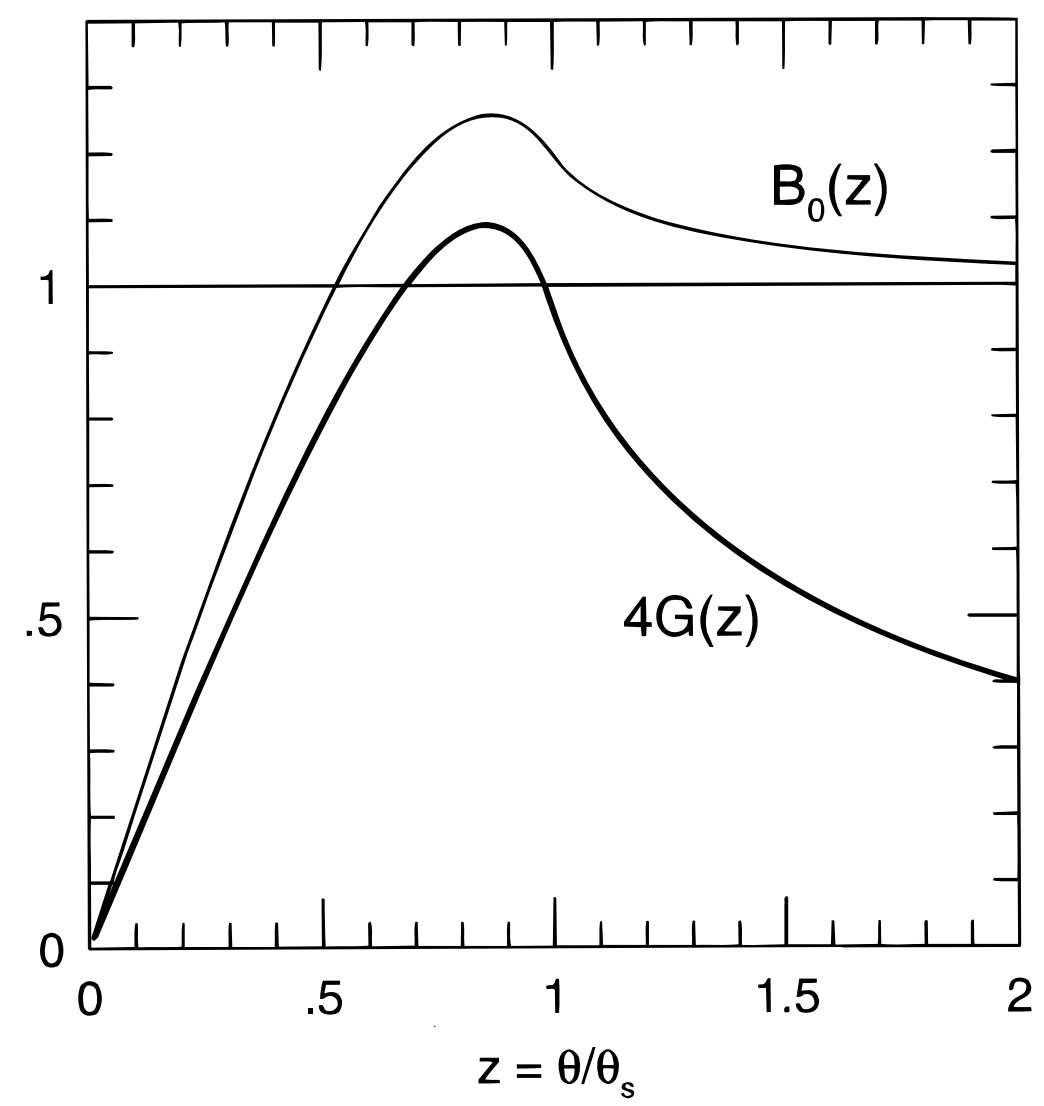

FIG. 1.-Deviation from point-source behavior as a function of $z$, the lens-source separation in units of the source size. Solid line: The magnification adjustment factor $B_{0}(z)$, which should be multiplied by the naive point-source magnification $A(x)$ to give the true magnification. Bold line: The velocity shift factor $G(z)$ (times four). The apparent shift in radial velocity is given by $v \sin i \sin \gamma G(z)$, where $v \sin i$ is the projected rotation speed and $\gamma$ is the angle between the projected source axis and the position of the lens. Note that the two functions are nearly identical for $z \lesssim 1$. 
The finite size of the source also induces a shift in the center of the spectral lines. Let $\gamma$ be the angle between the projected axis of source rotation and the position of the lens. Then the line shift is given by

$$
\Delta v(z, \gamma)=\frac{\int_{0}^{1} d z^{\prime} z^{\prime} S\left(z^{\prime}\right) \int_{0}^{2 \pi} d \phi A\left(q x_{*}\right)\left(z^{\prime} \sin \phi v \sin i\right)}{\int_{0}^{1} d z^{\prime} z^{\prime} S\left(z^{\prime}\right) \int_{0}^{2 \pi} d \phi A\left(q x_{*}\right)},
$$

where $\left(z^{\prime}, \phi\right)$ is the position on the star relative to the projected spin axis and $q^{2} \equiv z^{2}+z^{\prime 2}-2 z z^{\prime} \cos (\phi-\gamma)$. Assuming $x_{*} \ll 1$, this equation becomes

$$
\Delta v(z, \gamma)=v \sin i \sin \gamma G(z), \quad G(z) \equiv \frac{B_{1}(z)}{B_{0}(z)},
$$

where

$$
B_{n} \equiv \frac{\int_{0}^{1} d z^{\prime} z^{\prime} S\left(z^{\prime}\right) \int_{0}^{2 \pi} d \psi\left[1+\left(z^{\prime} / z\right)^{2}-2\left(z^{\prime} / z\right) \cos \psi\right]^{-1 / 2}\left(z^{\prime} \cos \psi\right)^{n}}{2 \pi \int_{0}^{1} d z^{\prime} z^{\prime} S\left(z^{\prime}\right)}
$$

and $\psi=\gamma-\phi$. The function $G(z)$ is shown in Figure 1.

The source-lens separation is given by

$$
x(t)=\left[\omega^{2}\left(t-t_{0}\right)^{2}+\beta^{2}\right]^{1 / 2},
$$

where $t_{0}$ is the time of maximum magnification, $\beta$ is the impact parameter in units of $\theta_{\mathrm{E}}$, and $\omega^{-1}$ is the Einstein-radius crossing time. Let $\alpha$ be the angle between the lens direction of motion and the projected spin axis of the source. Then equation (2.4) can be written

$$
\Delta v(t)=v \sin i \frac{\beta \cos \alpha+\omega\left(t-t_{0}\right) \sin \alpha}{x(t)} G\left[\frac{x(t)}{x_{*}}\right] .
$$

For giant-star events with low impact parameter $(\beta \ll 1)$, the parameters $t_{0}, \beta$, and $\omega$ are usually determined to better than $1 \%$ from the overall light curve, implying that $x(t)$ is also well determined. Hence, equation (2.7) is effectively a function of three parameters, $v \sin i, \alpha$, and $x_{*}$. In principle, one could fit the time series of observed line shifts for these three parameters. In practice, for events with $\beta \lesssim x_{*}$, it is much easier to measure $x_{*}$ by fitting photometric measurements to $A(x) B_{0}\left[x(t) / x_{*}\right]$. Hence, $x_{*}$ may also be regarded as a known quantity in equation (2.7), implying that $\Delta v$ is effectively a function of just two parameters, $v \sin i$ and $\alpha$.

To connect this work with the work of Maoz \& Gould (1994), I note that for $\beta \gtrsim x_{*}, G(z) \rightarrow \xi /(4 z)$, where $\xi=2 \int d z z^{3} S(z) /$ $\int d z z S(z)$, with $\xi \sim 0.89$ for the $I$ band. Thus $\Delta v \propto x_{*} v \sin i$, so that it is impossible to find $x_{*}$ and $v$ sin $i$ separately. Hence, unless the lens transits (or nearly transits) the source, or there is some other information about $x_{*}$ (see $\S 4$ ), one cannot use spectroscopy of lensing events to determine $v \sin i$.

Equation (2.7) was obtained under the assumptions $x_{*} \ll 1$ and $x \ll 1$. If one drops the latter assumption and repeats the derivation beginning with equation (2.3), one finds that equation (2.7) remains valid provided one makes the substitution

$$
G\left(\frac{x}{x_{*}}\right) \rightarrow \tilde{G}\left(x, x_{*}\right) \equiv G\left(\frac{x}{x_{*}}\right)\left[-x \frac{d \ln A}{d x}\right]=\frac{G\left(x / x_{*}\right)}{\left(1+x^{2} / 2\right)\left(1+x^{2} / 4\right)} .
$$

\section{ERROR ANALYSIS}

Suppose that a series of measurements $u_{k}$ are made of the apparent radial velocity of the star at times $t_{k}$ with errors $\sigma_{k}$. One then fits the measurements to a function of form

$$
F\left(t ; v_{0}, \eta, \alpha, v \sin i\right)=v_{0}+\eta t+\Delta v(\alpha, v \sin i),
$$

where $v_{0}$ is the true velocity of the source at the peak of the event, $\eta$ is the radial acceleration of the source, and $\Delta v(\alpha, v \sin i)$ is given by equation (2.7). Then one can estimate the covariances $c_{i j}$ of the determinations of the parameters $\left(a_{1}, a_{2}, a_{3}, a_{4}\right)=\left(v_{0}\right.$, $\eta, \alpha, v \sin i$ ) by (e.g., Gould 1995a),

$$
c=b^{-1}, \quad b_{i j}=\sum_{k} \sigma_{k}^{-2} \frac{\partial F\left(t_{k}\right)}{\partial a_{i}} \frac{\partial F\left(t_{k}\right)}{\partial a_{i}} .
$$

Of primary interest is $c_{4,4}^{1 / 2}$, the error in $v \sin i$.

In general, the errors will depend on the details of the experimental setup and on the observing conditions. In order to illustrate the overall sensitivity of the observations, I adopt a specific model. I assume that measurements are made at a rate $20 \omega / x_{*}$, that is, 20 times per source-radius crossing time. For typical events seen toward the bulge, $x_{*} / \omega \sim 10 \mathrm{hr}\left(r_{s} / 10 r_{\odot}\right)$, where $r_{s} / r_{\odot}$ is the radius of the source in solar units. I define $\delta v$ as the error at the peak of the event and assume that for other measurements, the errors scale inversely as the square root of the magnification. That is, $\sigma_{i}\left[x\left(t_{i}\right)\right]=$ $\delta v\left[B_{0}\left(\beta / x_{*}\right) x / B_{0}\left(x / x_{*}\right) \beta\right]^{1 / 2}$. I assume that the measurements are carried out from two source crossing times $\left(2 x_{*} / \omega\right)$ before the peak until five crossing times after the peak. The results expressed in units of $\delta v$ are shown in Table 1 for various values of $\alpha$ and $\beta / x_{*}$ (impact parameter in units of $\theta_{*}$ ). I have not shown the results for $\alpha>90^{\circ}$, which are almost exactly equal to the results for $180^{\circ}-\alpha$. 
TABLE 1

\begin{tabular}{|c|c|c|c|c|}
\hline \multirow[b]{2}{*}{$\beta / x_{*}$} & \multicolumn{4}{|c|}{ Position Angle $(\alpha)^{\mathbf{b}}$} \\
\hline & $0^{\circ}$ & $30^{\circ}$ & $60^{\circ}$ & $90^{\circ}$ \\
\hline $0.2 \ldots \ldots \ldots \ldots \ldots$ & 4.6 & 3.9 & 2.2 & 0.9 \\
\hline $0.4 \ldots \ldots \ldots \ldots$ & 2.3 & 1.9 & 1.2 & 1.0 \\
\hline $0.6 \ldots \ldots \ldots \ldots \ldots$ & 1.6 & 1.4 & 1.1 & 1.1 \\
\hline $0.8 \ldots \ldots \ldots \ldots \ldots$ & 1.4 & 1.2 & 1.3 & 1.4 \\
\hline $1.0 \ldots \ldots \ldots \ldots \ldots$ & 1.7 & 1.5 & 1.6 & 1.9 \\
\hline $1.2 \ldots \ldots \ldots \ldots \ldots$ & 2.3 & 2.0 & 2.0 & 2.4 \\
\hline $1.4 \ldots \ldots \ldots \ldots \ldots$ & 3.0 & 2.4 & 2.4 & 3.0 \\
\hline
\end{tabular}

From Table 1, one sees that, for example, with radial velocity errors of $\delta v \sim 0.1 \mathrm{~km} \mathrm{~s}^{-1}$ one could typically expect to measure $v \sin i$ to an accuracy of $0.2-0.3 \mathrm{~km} \mathrm{~s}^{-1}$, although the errors are larger for some unfavorable geometries.

Next I examine how sensitively these results depend on the continuous coverage assumed in the construction of Table 1 . In Table 2, I have assumed that observations are made for two out of every three crossing times. In Table 3, I assume that observations are made for one out of every three crossing times. The three tables then roughly approximate what could be done with three, two, and one observatories, respectively. In order to facilitate comparison with Table 1, I assume the same number of total observations. That is, observations are carried out at a rate $30 \omega / x_{*}$ for Table 2 and at $60 \omega / x_{*}$ for Table 3 . It is clear from these tables that organizing observations from several sites around the world has important advantages.

One possible method of further reducing the errors is to obtain independent information on the zero-point velocity $v_{0}$ and the acceleration $\eta$. Such constraints could, in principle, be obtained by observing the source after the event was over to look for periodic motion due to a companion. Unfortunately, as I now show, obtaining significant improvements on the errors reported above would be quite difficult. By examining the covariance matrix $c_{i j}$, I find that typically $\left(c_{2,2} / c_{4,4}\right)^{1 / 2} \sim 0.1 \omega / x_{*}$. Hence if, for example, $v \sin i$ were determined with accuracy $300 \mathrm{~m} \mathrm{~s}^{-1}$ and the crossing time were $\sim 0.5$ day, $\eta$ would be known with an accuracy of $60 \mathrm{~m} \mathrm{~s}^{-1}$ day $^{-1}$, about one-eighth the acceleration of the Earth. To obtain a significant improvement on the measurement of $v \sin i$ one would have to measure $\eta$ independently to at least this accuracy. However, such a small acceleration could be produced by, for example, a planet of 4 Jupiter masses at 0.2 AU. Such a planet would generate redshift oscillations with an amplitude of only $300 \mathrm{~m} \mathrm{~s}^{-1}$. These oscillations would be extremely difficult to detect.

TABLE 2

\begin{tabular}{|c|c|c|c|c|}
\hline \multirow[b]{2}{*}{$\beta / x_{*}$} & \multicolumn{4}{|c|}{ Position Angle $(\alpha)^{\mathrm{b}}$} \\
\hline & $0^{\circ}$ & $30^{\circ}$ & $60^{\circ}$ & $90^{\circ}$ \\
\hline $0.2 \ldots \ldots \ldots \ldots$ & 4.6 & 4.2 & 2.8 & 1.3 \\
\hline $0.4 \ldots \ldots \ldots \ldots$ & 2.3 & 2.3 & 1.9 & 1.4 \\
\hline $0.6 \ldots \ldots \ldots \ldots \ldots$ & 1.6 & 1.8 & 1.8 & 1.6 \\
\hline $0.8 \ldots \ldots \ldots \ldots$ & 1.4 & 1.7 & 1.9 & 1.9 \\
\hline $1.0 \ldots \ldots \ldots \ldots \ldots$ & 1.7 & 1.9 & 2.2 & 2.2 \\
\hline $1.2 \ldots \ldots \ldots \ldots \ldots$ & 2.3 & 2.3 & 2.4 & 2.5 \\
\hline $1.4 \ldots \ldots+\ldots, \ldots$ & 2.9 & 2.7 & 2.7 & 2.9 \\
\hline
\end{tabular}

${ }^{a}$ In units of measurement error $\delta v$.

${ }^{\mathrm{b}}$ Relative to the star rotation axis.

TABLE 3

“ONE OBSERVATORY” ERRORS IN $v \sin i^{\mathrm{a}}$

\begin{tabular}{crrrr}
\hline \hline & \multicolumn{4}{c}{ Position Angle $(\alpha)^{\mathrm{b}}$} \\
\cline { 2 - 5 }$\beta / x_{*}$ & \multicolumn{1}{c}{$0^{\circ}$} & \multicolumn{1}{c}{$30^{\circ}$} & $60^{\circ}$ & $90^{\circ}$ \\
\hline $0.2 \ldots \ldots \ldots \ldots \ldots$. & 14.3 & 12.0 & 6.4 & 1.4 \\
$0.4 \ldots \ldots \ldots \ldots \ldots \ldots$ & 6.4 & 5.1 & 2.6 & 1.3 \\
$0.6 \ldots \ldots \ldots \ldots \ldots \ldots$ & 3.7 & 2.9 & 1.6 & 1.4 \\
$0.8 \ldots \ldots \ldots \ldots \ldots \ldots$ & 2.7 & 2.1 & 1.5 & 1.8 \\
$1.0 \ldots \ldots \ldots \ldots \ldots \ldots$ & 3.2 & 2.5 & 2.1 & 2.5 \\
$1.2 \ldots \ldots \ldots \ldots \ldots \ldots$ & 5.1 & 3.8 & 2.8 & 3.5 \\
$1.4 \ldots \ldots \ldots \ldots \ldots$. & 7.1 & 5.2 & 3.7 & 4.8 \\
\hline
\end{tabular}

${ }^{\mathrm{a}}$ In units of measurement error $\delta v$.

${ }^{\mathrm{b}}$ Relative to the star rotation axis. 
The main reason that one can hope to measure subtle changes in apparent redshift during the lensing event is that the star is magnified $\gtrsim 10$ times. After the event is over and the star returns to its unlensed luminosity, typically $I \lesssim 16$, highly accurate velocity measurements become extremely difficult.

\section{DISCUSSION}

In the analysis given in $\S 2, \mathrm{I}$ implicitly assumed that the local strength of the lines used to measure the redshift is proportional to the local surface brightness. In fact, since limb darkening is caused in part by line absorption, one expects that the line strength is more heavily weighted toward the limb of the star than the luminosity. This fact poses no fundamental difficulties, since one can simply replace $S(z)$ with the line-strength density in equation (2.3) and the following discussion. The line strength and $S(z)$ are calculated in the same stellar-atmosphere codes. However, the complication does break the purely empirical link between the determination of $x_{*}$ from photometry (which depends only on $S$ ), and the use of $x_{*}$ in the interpretation of the lineshift data. This link is illustrated by the similar forms of $B_{0}$ and $G$ for $z \lesssim 1$ in Figure 1 . For most cases the systematic errors induced by this effect will be small.

I now briefly describe several possible extensions of the basic method presented in this paper. The most straightforward extension is to use the Ca II line at $393.3 \mathrm{~nm}$ to measure the line shift. Loeb \& Sasselov (1995) showed that narrow-band imaging of this line during a lensing event could be used to measure $x_{*}$. Giant stars are limb-brightened in Ca II, so the surface brightness has a ringlike structure. The same feature would make it especially useful for monitoring lineshifts during the event. The formalism presented in $\S 2$ is easily adapted to this case by simply replacing $S(z)$ with the emission profile. If one uses the Loeb \& Sasselov (1995) photometric method for determining $x_{*}$ (either by measuring the absolute line fluxes from spectroscopy or by independent narrow-band imaging), systematic errors should be small. On the other hand, if $x_{*}$ were measured by some other means, the accuracy of the results would depend more critically on the accuracy of the model. A limitation of this method is that many bulge fields are heavily reddened. Since the extinction at $393 \mathrm{~nm}$ is $\sim 2.7$ times greater than that in the $I$ band, it should often be much easier to measure redshifts from cross-correlation in the $I$ band than from the Ca in line.

If the stellar radius could be measured for $z \gtrsim 1, v \sin i$ could be determined for a much larger number of giants, because for $z \gtrsim 1$, the lineshift falls off only as $\Delta v \propto z^{-1}$ (Maoz \& Gould 1994). One method of extending the measurement to $x_{*} \sim 2$ is optical/infrared photometry. In this method, the inferred value of $z$ is $\propto\left(\Lambda^{H}-\Lambda^{V}\right)^{-1}$, where $\Lambda$ is proportional to the second moment of the surface brightness in $V$ and $H$ (Gould \& Welch 1996). Since the modeling of this combination of parameters is relatively independent of the modeling of the line-strength profile, there is a somewhat greater possibility of systematic errors than there is for the method of $\S \S 2$ and 3.

Finally, it may be possible to use optical interferometry to directly image the two source images during a lensing event (Gould 1996). This method does not depend at all on the lens being near the face of the source. Direct imaging yields a measurement of $\theta_{\mathrm{E}}$ and also of the direction of motion (Gould 1992). If $\theta_{*}$ can be determined from Stefan's law, then $x_{*}=\theta_{*} / \theta_{\mathrm{E}}$ is determined. The image separation is $2 \theta_{\mathrm{E}}$, which typically is of order $100 \mu$ as. A highly magnified giant might have $I \sim 14$ mag. It will be at least a few years before such measurements are possible, but this method may permit the measurement of $v \sin i$ for a large number of giants. Recall that the lineshifts also measure $\alpha$, the angle between the projected spin axis and the direction of lens motion. Since optical interferometry measures the absolute direction of lens motion, one can also obtain the absolute direction of the projected spin axis.

I would like to thank M. Pinsonneault for stimulating discussions. This work was supported in part by grant AST 94-20746 from the NSF.

\section{REFERENCES}

Alard, C. 1996, in IAU Symp. 173, Astrophysical Applications of Gravitational Lensing, ed. C. S. Kochanek \& J. N. Hewitt (Dordrecht: Kluwer), 215

Alcock, C., et al. 1995, ApJ, 445, 133

Ansari, R., et al. 1996, A\&A, 314, 94

Aubourg, E., et al. 1993, Nature, 365, 623

Gould, A. 1992, ApJ, 392, 442 1994, ApJ, 421, L71

1995a, ApJ, 440, 510

.1995b, ApJ, 447, 491

Gould, A. 1996, PASP, 108, 465

Gould, A., \& Welch, D. L. 1996, ApJ, 464, 212

Gray, D. F. 1989, ApJ, 347, 1021

Loeb, A., \& Sasselov, D. 1995, ApJ, 449, L33

Maoz, D., \& Gould, A. 1994, ApJ, 425, L67

Nemiroff, R. J., \& Wickramasinghe, W. A. D. T. 1994, ApJ, 424, L21

Olech, A. 1997, Acta Astron., in press

Udalski, A., et al. 1994, Acta Astron., 44, 165

Witt, H., \& Mao, S. 1994, ApJ, 430, 505 